Stratospheric Relaxation in

IMPACT's Radiation Code

T. Edis, K. Grant, P. Cameron-Smith

November 15, 2006 
This document was prepared as an account of work sponsored by an agency of the United States Government. Neither the United States Government nor the University of California nor any of their employees, makes any warranty, express or implied, or assumes any legal liability or responsibility for the accuracy, completeness, or usefulness of any information, apparatus, product, or process disclosed, or represents that its use would not infringe privately owned rights. Reference herein to any specific commercial product, process, or service by trade name, trademark, manufacturer, or otherwise, does not necessarily constitute or imply its endorsement, recommendation, or favoring by the United States Government or the University of California. The views and opinions of authors expressed herein do not necessarily state or reflect those of the United States Government or the University of California, and shall not be used for advertising or product endorsement purposes.

This work was performed under the auspices of the U.S. Department of Energy by University of California, Lawrence Livermore National Laboratory under Contract W-7405-Eng-48. 


\title{
Stratospheric Relaxation in IMPACT's Radiation Code
}

\author{
Taner Edis, Keith Eric Grant, Philip Cameron-Smith
}

November 13, 2006

While IMPACT incorporates diagnostic radiation routines from our work in previous years, it has not previously included the stratospheric relaxation required for forcing calculations. We have now implemented the necessary changes for stratospheric relaxation, tested its stability, and compared the results with stratosphere temperatures obtained from CAM3 met data.

The relaxation results in stable temperature profiles in the stratosphere, which is encouraging for use in forcing calculations. It does, however, produce a cooling bias when compared to CAM3, which appears to be due to differences in radiation calculations rather than the interactive treatment of ozone. The cause of this bias is unclear as yet, but seems to be systematic and hence cancels out when differences are taken relative to a control simulation.

\section{Temperature Perturbations}

We have adjusted the stratospheric temperature by working with the potential temperature $\theta$, which is conserved when an air mass is subjected to adiabatic changes: ${ }^{1}$

$$
\theta=T\left(\frac{p_{0}}{p}\right)^{\kappa}
$$

where the reference temperature $p_{0}$ is conventionally taken as $1000 \mathrm{mb}$, and $\kappa=R / C_{p}$. We take $\kappa=0.285572139$, the value for dry air-it does not

\footnotetext{
${ }^{1}$ David G. Andrews, An Introduction to Atmospheric Physics (Cambridge, UK: Cambridge University Press, 2000), pp. 28-29.
} 
change significantly for our purposes.

Within the context of a chemical-radiative-transport model, we can define a stratospheric radiative-advective balance for potential temperature through an equation setting the advective derivative of $\theta$ equal to a total heating rate. Potential temperature, in a sense, can be considered as the mixing ratio for sensible heat:

$$
\frac{\partial \theta}{\partial t}+\vec{v} \cdot \vec{\nabla} \theta=Q_{\mathrm{rad}}+Q_{\mathrm{app}}
$$

In this equation, the velocity's dot product with the gradient of potential temperature captures the advection. $Q_{\mathrm{rad}}$ is the scaled total radiative heating rate,

$$
Q_{\mathrm{rad}}=\left(Q_{\mathrm{sw}}+Q_{\mathrm{lw}}\right)\left(\frac{p_{0}}{p}\right)^{\kappa}
$$

where $Q_{\mathrm{sw}}$ and $Q_{\mathrm{lw}}$ are the shortwave and longwave heating rates. These are normally calculated within IMPACT's radiation routines. $Q_{\text {app }}$ is an apparent source or sink of heating required to balance the equation in the unperturbed atmosphere for prescribed $\theta$. It is the net effect of all the errors from calculating the other terms.

For a chemically perturbed atmosphere, the radiative heating will change. The extra term $Q_{\text {app }}$ makes (2) difficult to solve. If we can assume that $Q_{\text {app }}$ is approximately the same in the perturbed and unperturbed cases, then the difference in temperature can be obtained, which is what is needed for forcing calculations. In that case, we work with the equation

$$
\frac{\partial \theta}{\partial t}+\vec{v} \cdot \vec{\nabla} \theta=Q_{\mathrm{rad}}
$$

in order to calculate the stratosphere temperature for the radiation routines within IMPACT. We expect the relaxation times for the stratosphere to be of the order of a day, which is much larger than the time step of IMPACT, typically about an hour. Therefore a simple linear approximation, where

$$
\Delta \theta=\left(Q_{\mathrm{rad}}-\vec{v} \cdot \vec{\nabla} \theta\right) \Delta t
$$

with $\Delta t$ the IMPACT time step, should quickly converge onto a stable value for the temperature. 


\section{Implementation}

IMPACT uses an operator-split technique, handling processes such as advection, chemistry, radiation etc. in a given sequence. Therefore the advective and radiative terms in (5) will be calculated in the appropriate places in each time step.

The advective contribution to the potential temperature change in (5) can be computed by the usual IMPACT routine for advection, if $\theta$ is treated in the same way as a tracer species. So the routine Update_Advec in source file advec_update.F was modified to receive a $3 \mathrm{D}$ potential temperature array as a parameter, and advect it just like a tracer species.

The radiation operator follows later in each time step. Here, the radiation is calculated with an air temperature derived from the advected potential temperature, and not the variable kel obtained from the met data as was the previous practice in the radiation routines. This required appropriate modifications to $C a l c_{-}$Radiation in radiation.F.

Finally, (3) is used to get the radiative change in $\theta$ due to the calculate longwave and shortwave heating rates. This change is imposed in the stratosphere only - where the pressure is less than the tropopause pressure tropp already calculated in IMPACT. The troposphere temperature is set equal to kel, discarding all changes. We considered relaxing the tropospheric temperature toward kel more gradually, but that created spurious artifacts in the troposphere and testing showed that immediate adjustment was feasible, so that was finally implemented.

Since radiation is a function of temperature rather than potential temperature, our primary variable (kel_rad) contains the air temperature for the radiation. This is converted to potential temperature for advection and converted back afterwards.

The basic time step routine, Imp_Step in imp_step.F, was modified in order to handle the advective and radiative changes calculated for kel_rad. In addition, the new source file potential_temp. $F$ was added to the radiation package; it contains a number of potential temperature-related routines.

Implementing stratospheric relaxation also required a number of new inputs and outputs for IMPACT. There are two new namelist variables, do_kel_rad and pr_kel_rad. These instruct IMPACT to do the potential temperature calculations and to output the results as part of the general radiation output in the .rad.nc file. Both are F (false) by default.

The stratospheric relaxation related output involves the variables kel_rad, 
htrt_adv, and htrt_rad - the air temperature $(\mathrm{K})$, the heating rate due to the advection $(\mathrm{K} /$ day), and the heating rate due to radiation $(\mathrm{K} /$ day). As with other radiation-related variables output by IMPACT, these are all mean values. Note that all three are 4D variables: 3D plus the additional radiation dimension that refers to the number of radiation calculations done. The user has the option of doing a number of radiation calculations within the same run, for example, turning on and off various combinations of aerosol species. Each radiation calculation will have its own separate potential temperature.

The input and output related routines of IMPACT were altered to handle the new variables. Additionally, kel_rad had to be recorded in the restart file to allow for long runs. Therefore the restart-related files were also modified.

All these changes to IMPACT were based on the latest version, archived on 6/23/2006. /home/edis/Impact06/gem-changes.tar on the ASD computer system contains the new and modified code. This can be untarred in the parent directory of gem/ to be incorporated into IMPACT before these changes are archived.

\section{A Colder Stratosphere}

When starting from scratch, kel_rad is initialized to equal kel from the met data. After a model time of about one week, long time runs settle into a stable temperature pattern in the stratosphere, as recorded in kel_rad. This is a positive result, as it suggests that the neglected term $Q_{\text {app }}$ in (2) is stable, and that looking for differences in forcing calculations will be meaningful. See figure 1, generated for an IMPACT run with TS5 chemistry and CAM3 met data, starting at the beginning of January and going on for just over 6 months.

While the relaxed stratospheric temperature shows a stable pattern, it is consistently cooler than the temperatures obtained from the CAM3 met data. Figure 2 shows the difference between kel_rad (the relaxed temperature derived from $\theta$ ) and kel (the met data temperature that is used in the chemistry), for the same days as in figure 1 . The stratosphere is about $10 \mathrm{~K}$ cooler compared to the met data. And the difference is particularly pronounced in the topmost layer, though the polar regions also tend to produce larger differences.

Plots of the contributions to the heating due to the advection and radiation (recall equation 4) appear in figure 3. In the stratosphere, the con- 
tribution from the radiation shows a distinct pattern of polar cooling and equatorial heating, while the tendency of the advective contribution is the opposite, as expected. In both cases, the polar regions tend to produce some very large values.

While it could not be expected that kel and kel_rad would match closely, especially since $Q_{\text {app }}$ has been neglected in equation (4), the $\sim 10 \mathrm{~K}$ cooling bias needs to be understood better. We investigated a few possible causes, including the role of ozone and how the top layer of the atmosphere is handled.

\section{Tropospheric Ozone}

Since IMPACT does ozone chemistry while CAM3 relies on an input ozone profile that is a zonal-mean monthly-mean, one contribution to the cooling bias might come through the ozone. In addition, it is also of independent interest to study where in the atmosphere the temperature is most sensitive to tropospheric ozone - most probably the tropopause.

To begin investigating such questions, we performed three short runstwo days of model time. This captures the beginning of stratospheric relaxation from the initial temperature profile, and two days is comparable to the relaxation times expected for the radiative contribution. One run was the normal IMPACT run, now just run for two days with hourly output. The other two runs replaced IMPACT's ozone treatment with CAM3 ozone processed to serve as a "fixed species" for IMPACT. One of these was with normal CAM3 ozone, and the other with the same ozone but with all ozone in the troposphere (below $150 \mathrm{ppb}$ ozone) set to zero.

There is no greatly significant difference between the results of these runs. The difference between kel_rad and kel at the end of 48 hours looks very similar in all three cases; for example, figure 4 . In the bottom graph, the difference between kel_rad - kel values is plotted, with the difference between the differences from the full ozone and zero tropospheric ozone runs with the ozone taken from CAM3 and held fixed. Tropospheric ozone is clearly leading to heating at the tropopause and cooling in the equatorial stratosphere, as expected.

The tendency seen in figures 4 and 5 motivates a closer look at the heating rates calculated by the radiation code. In figure 6 , the plots show the

differences between the total heating rate for the full-ozone case and the 
total heating rate for the zero tropospheric ozone case. There is indeed a slight tendency for stratospheric cooling and heating at the tropopause due to ozone in the troposphere. And as the lower plot in figure 6 displaying the heating rate difference after 48 hours have elapsed indicates, there is a moderation in these tendencies as the stratospheric relaxation takes hold. For further emphasis, we can plot the difference between the heating rate differences shown in figure 6 top and bottom, and also their ratios. These comparisons appear in figure 7.

We also did a pair of runs with the CAM3 model (not IMPACT, as all the previous runs) in order to generate CAM3 heating rates for the same time of year - the first two days of January. As before, the two runs were distinguished by the presence or absence of tropospheric ozone. The differences between the longwave and shortwave heating rates in these runs are plotted in figure 8, comparing the end of the two day runs. As expected, tropospheric ozone primarily affects the longwave heating. The noise in the troposphere is due to changing weather conditions; it can be disregarded.

Figure 9 shows the difference between the total heating rates of the runs with tropospheric ozone and without, for the first meaningful output record (day 1, hour 1:30) and the last (day 2, hour 23:30). As expected, tropospheric ozone leads to cooling in the equatorial stratosphere and heating in the upper troposphere.

As a final comparison, figure 10 shows the difference between the temperature responses of the IMPACT and CAM3 models. It plots the difference between the heating rate differences (between with and without ozone cases) in IMPACT and CAM3. The overall discrepancy in effect is small, as tropospheric ozone has similar effects on heating rates in both cases. This validates the relaxation capability we added to IMPACT and confirms that the $Q_{\text {app }}$ term in equation (2) cancels sufficiently for the results to be useful.

While the effect of ozone is similar in how CAM3 and IMPACT treat radiation, it is also clear that the $\sim 10 \mathrm{~K}$ bias found in the stratosphere is not due to ozone. The broad similarity in trend suggests that neglecting $Q_{\text {app }}$ in equation (4) will not affect the differences needed for forcing calculations. A better test might be to obtain a long $(\sim 10$ year $)$ run to allow kel_rad to adjust according to the advective-relaxation time scale, which is of the order of years. Such a run need not be prohibitively expensive, as chemistry etc. can be turned off and only the radiation and advection calculations need take place. Unfortunately, although we tried some quick modifications to allow such a run, it appears that radiation calculations in such stripped- 
down circumstances are not immediately feasible.

\section{The Top Layer}

In figures 2 and 4 , it is clear that the top layer of the atmosphere behaves somewhat anomalously in IMPACT calculations. We ran a quick test to make sure that the $\sim 10 \mathrm{~K}$ bias was not due to the top layer - a month-long run in which the top layer heating rates were set equal to the heating rates in the layer just below.

The results are shown in figure 11. The top plot is kel_rad - kel for this run, showing that although slightly smaller, the bias remains. The bottom plot is the difference between the bias in the top plot and the equivalent for the the same day with the unaltered radiation routines. Clearly the difference is small, so most of the bias is not due to the treatment of the top of the atmosphere.

\section{Conclusion}

Stratospheric relaxation is a necessary component of the radiation code in IMPACT if it is to be used in forcing calculations. We have implemented this relaxation and tested it. Our results indicate that it is stable, and that it produces behavior in line with qualitative expectations. The cooling bias when compared to CAM3 is the major outstanding question that remains; however, the existence of a bias is not very surprising. It is very likely that this is due to differences in how radiation is calculated in two models. In any case, our tests indicate that the bias largely cancels out when calculating the differences in fields relative to a control case, which will be the main use of this stratospheric relaxation capability. 

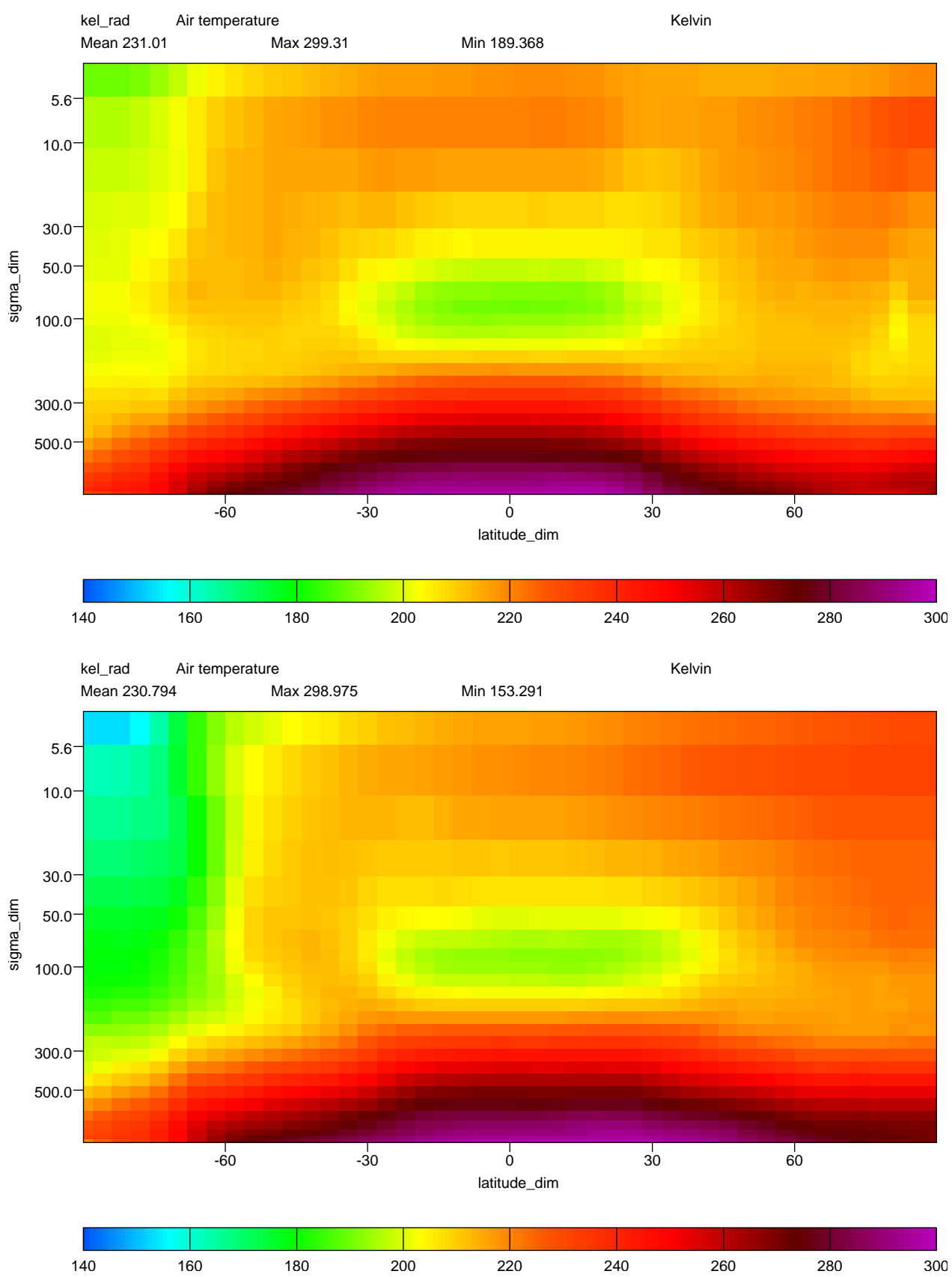

Figure 1: Zonal averages of kel_rad (K), mean values for day 97 and day 185. 

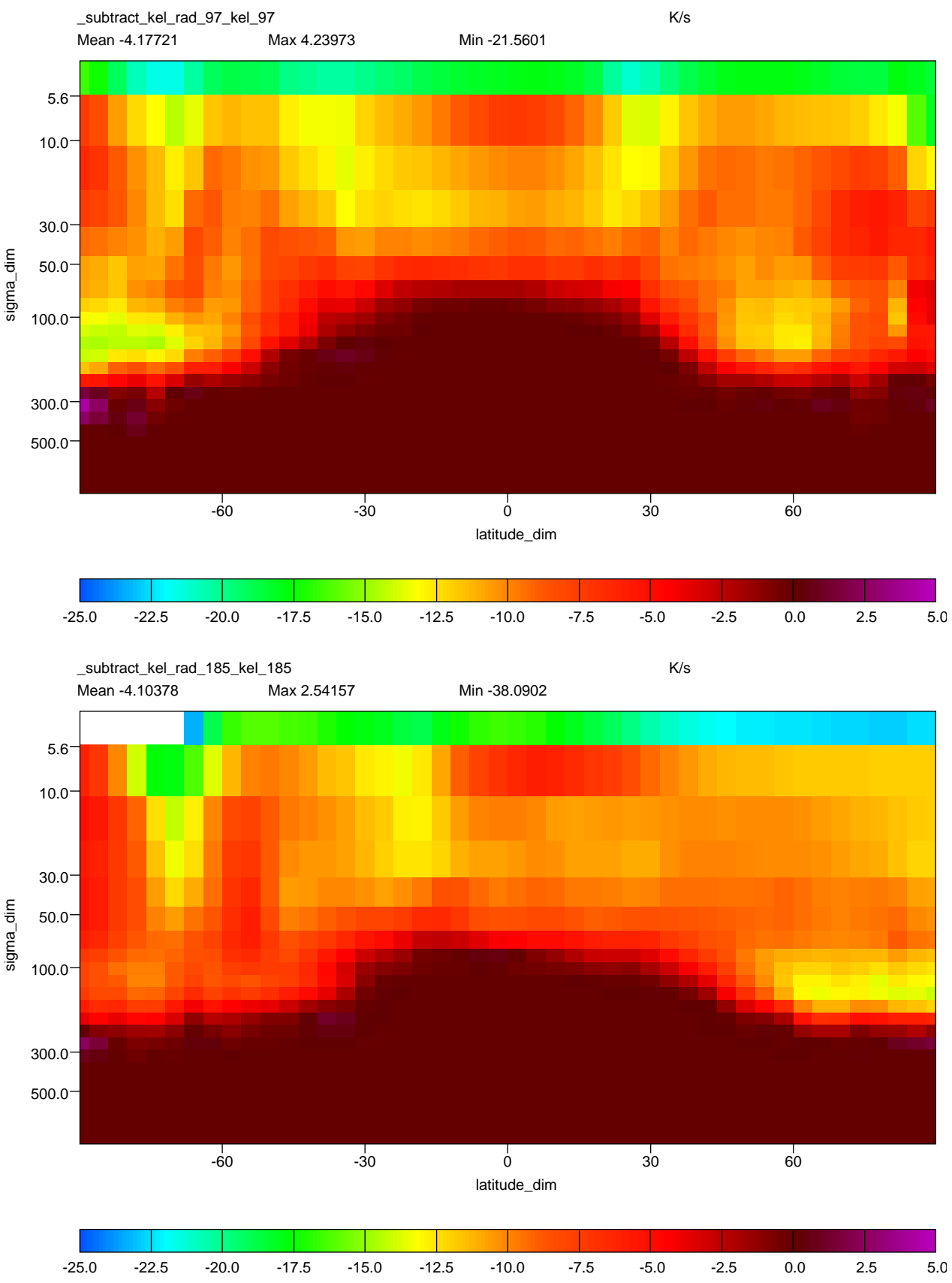

Figure 2: Zonal averages of kel_rad - kel ( $\mathrm{K}-\mathrm{K} / \mathrm{s}$ is due to a vcdat bug), mean values for day 97 and day 185 . Note the consistently cooler kel_rad in the stratosphere. 

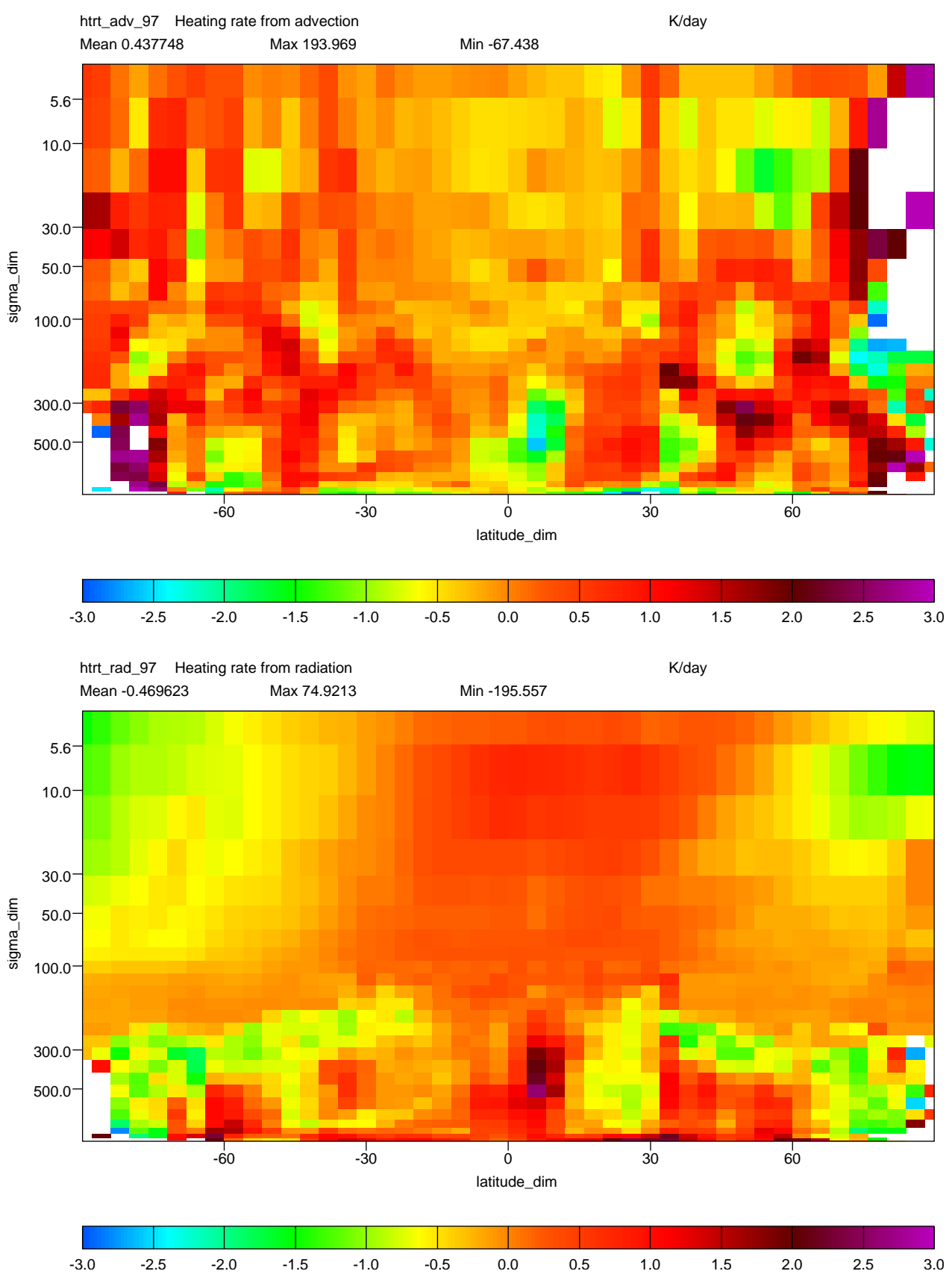

Figure 3: Zonal averages of the heating rates due to advection (top) and radiation (bottom) (K/day), mean values for day 97 . White spaces are extreme values at the poles. 


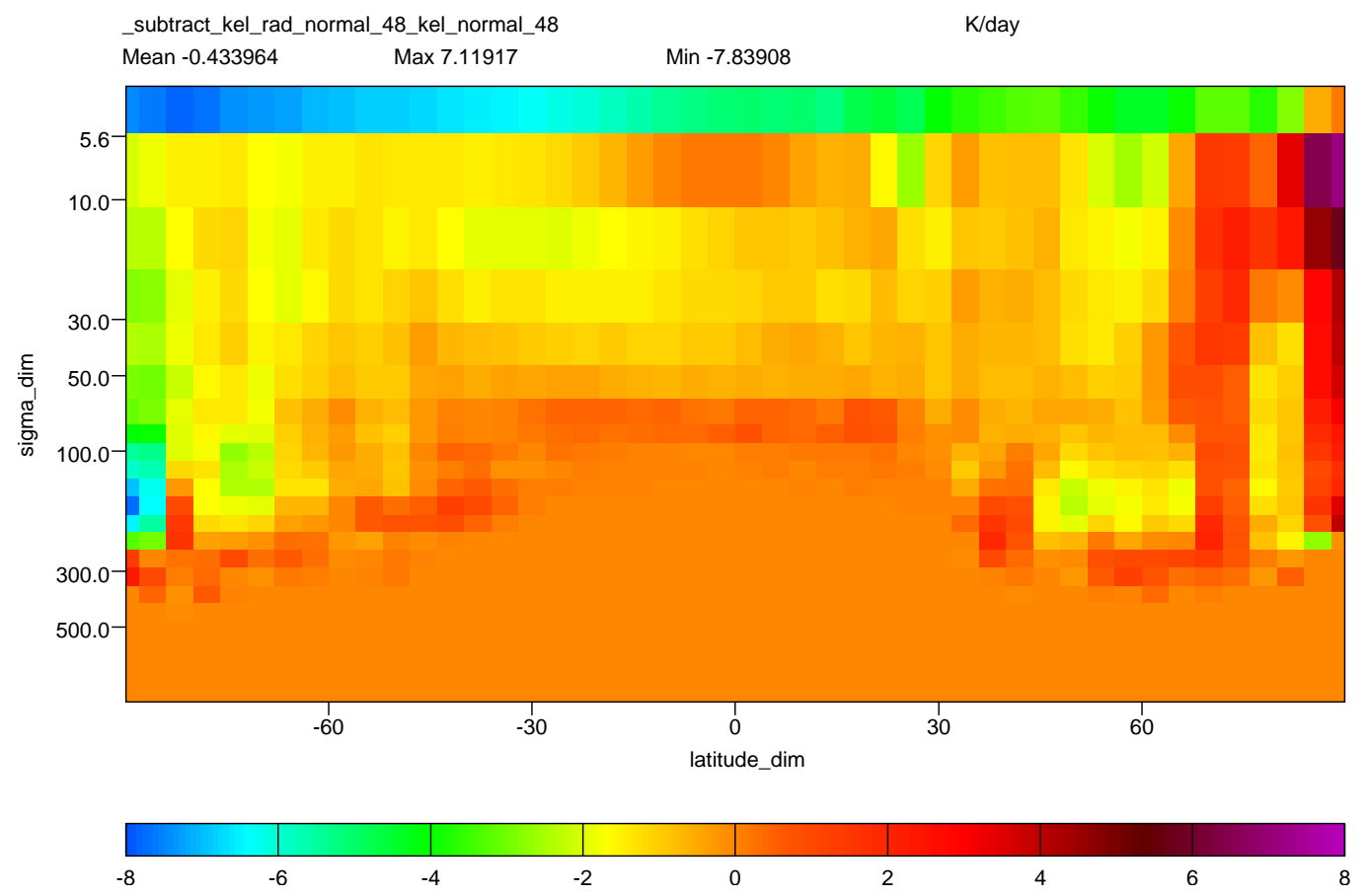

Figure 4: Zonal average of kel_rad - kel (the difference between the temperature calculated for the stratospheric relaxation and that used for the chemistry calculations) after 48 hours, with normal IMPACT ozone. 


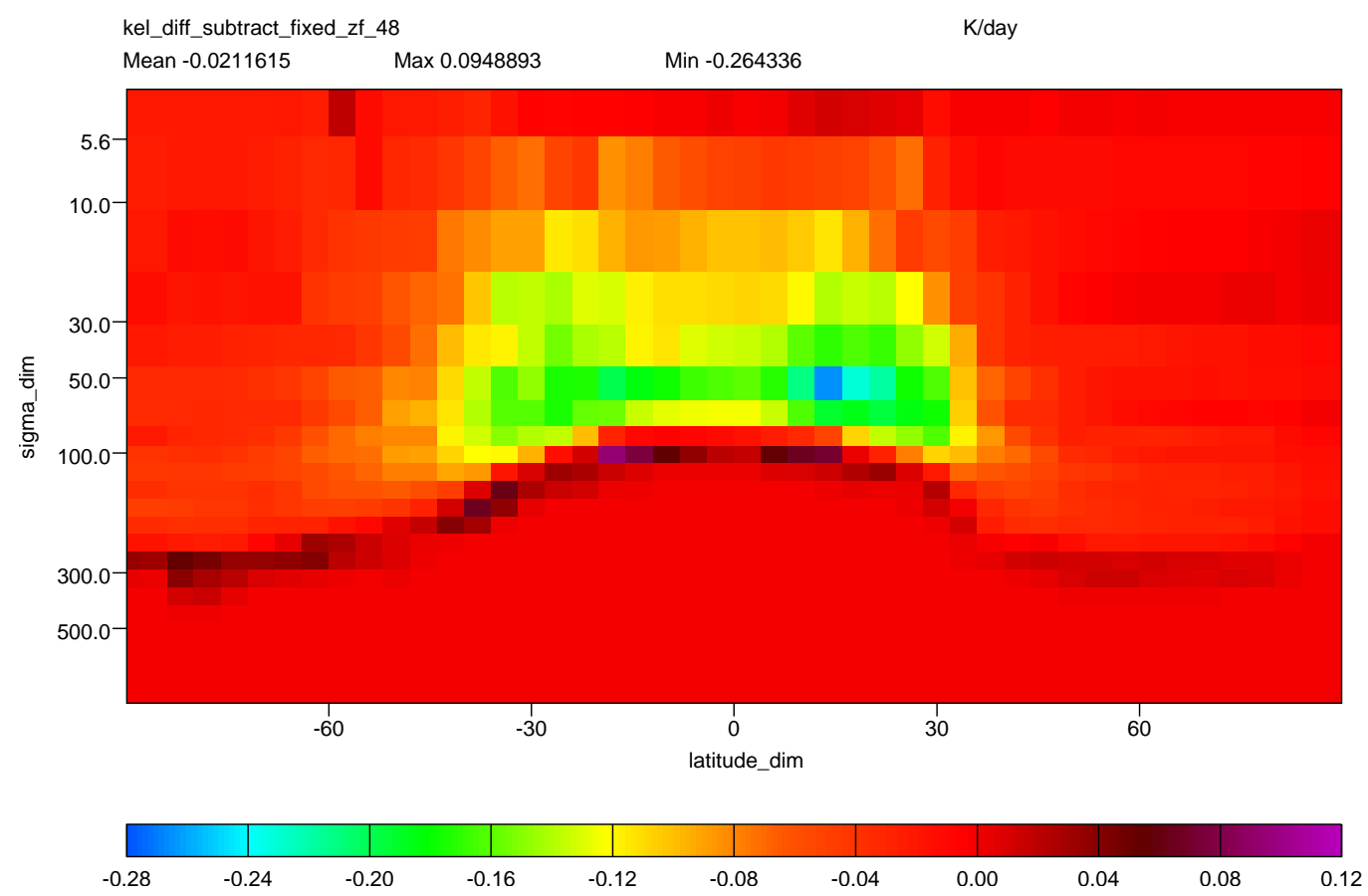

Figure 5: The temperature difference kel_rad - kel was calculated for two cases: IMPACT run with CAM3 ozone, and IMPACT run with an ozone profile derived from CAM3 by setting tropospheric ozone to zero. The graph displays the difference between the kel_rad - kel values calculated for the full ozone and the zeroed ozone cases. It shows that tropospheric ozone leads to the expected cooling in the equatorial stratosphere. 

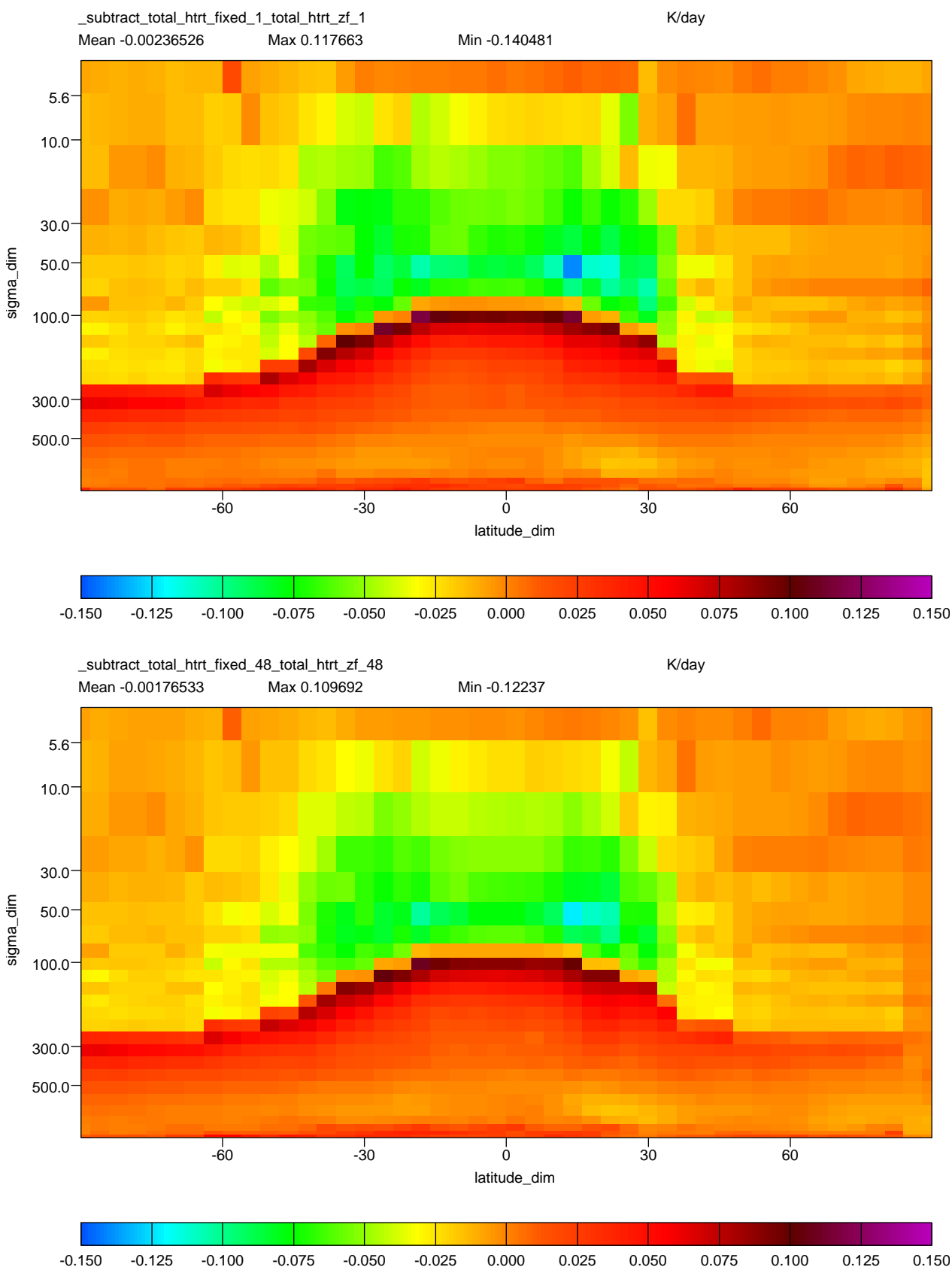

Figure 6: The difference between the total heating rates calculated for the full CAM3 ozone and zero tropospheric CAM3 ozone cases, after hour 1 (top) and hour 48(bottom). 

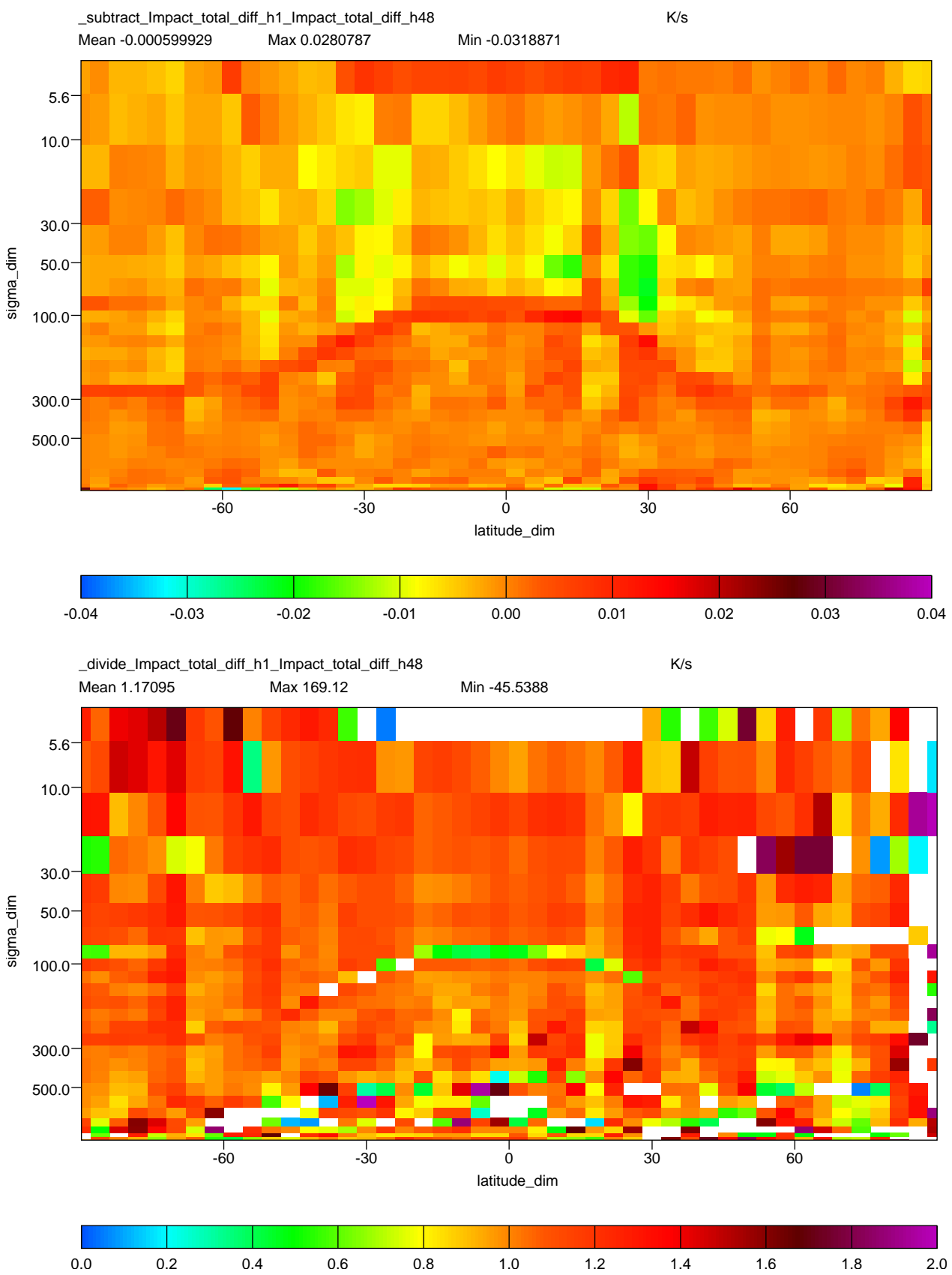

Figure 7: Top: The difference between the top and bottom plots in figure 6 (previous page). Bottom: Their ratios (the ozone after one hour divided by the ozone after 48 hours), with white space excluded extreme values. 

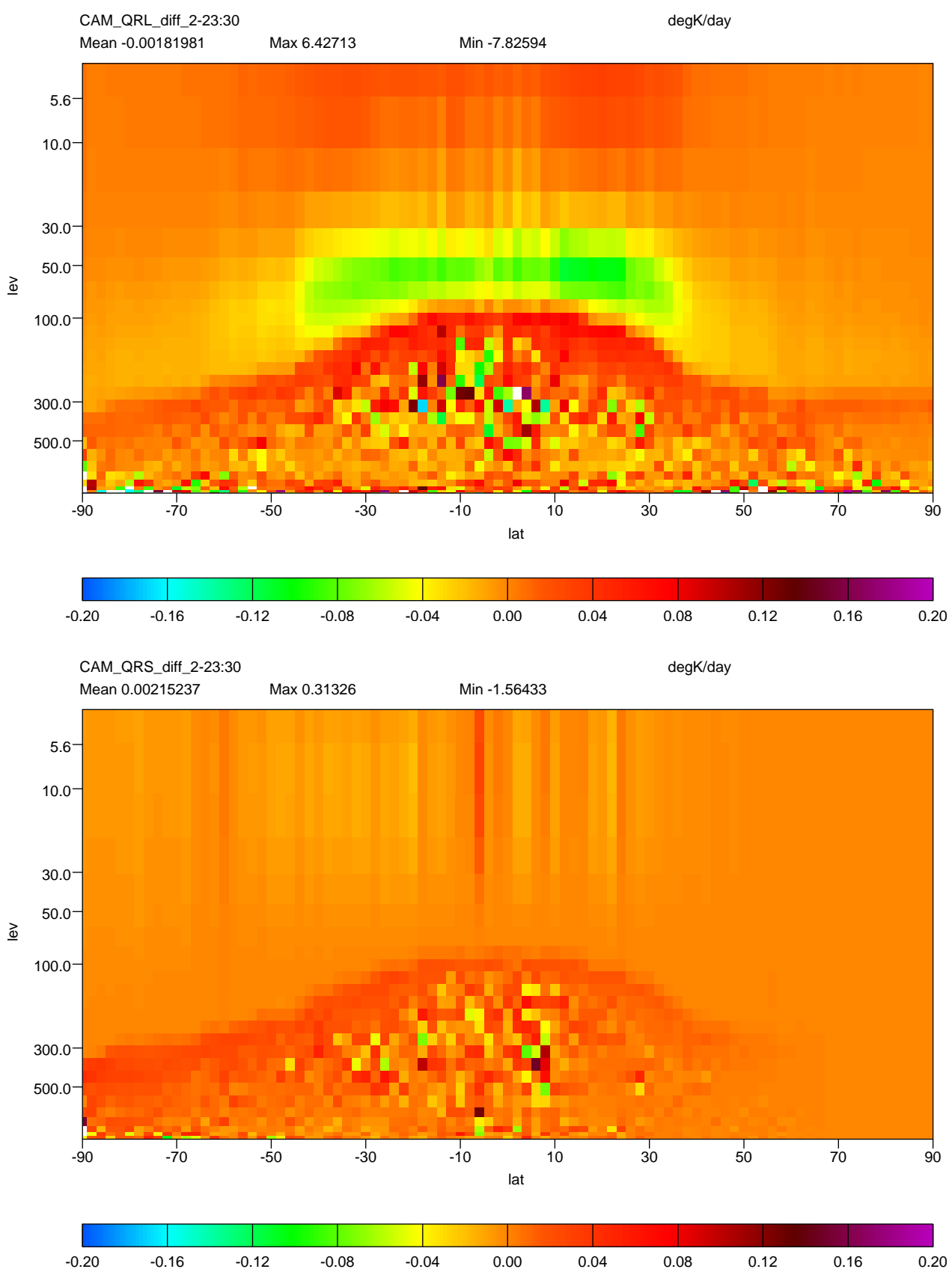

Figure 8: Difference between heating rates from the CAM3 model with tropospheric ozone and the CAM3 model without ozone in the longwave (top) and shortwave (bottom), for the last timestep of the second day. 

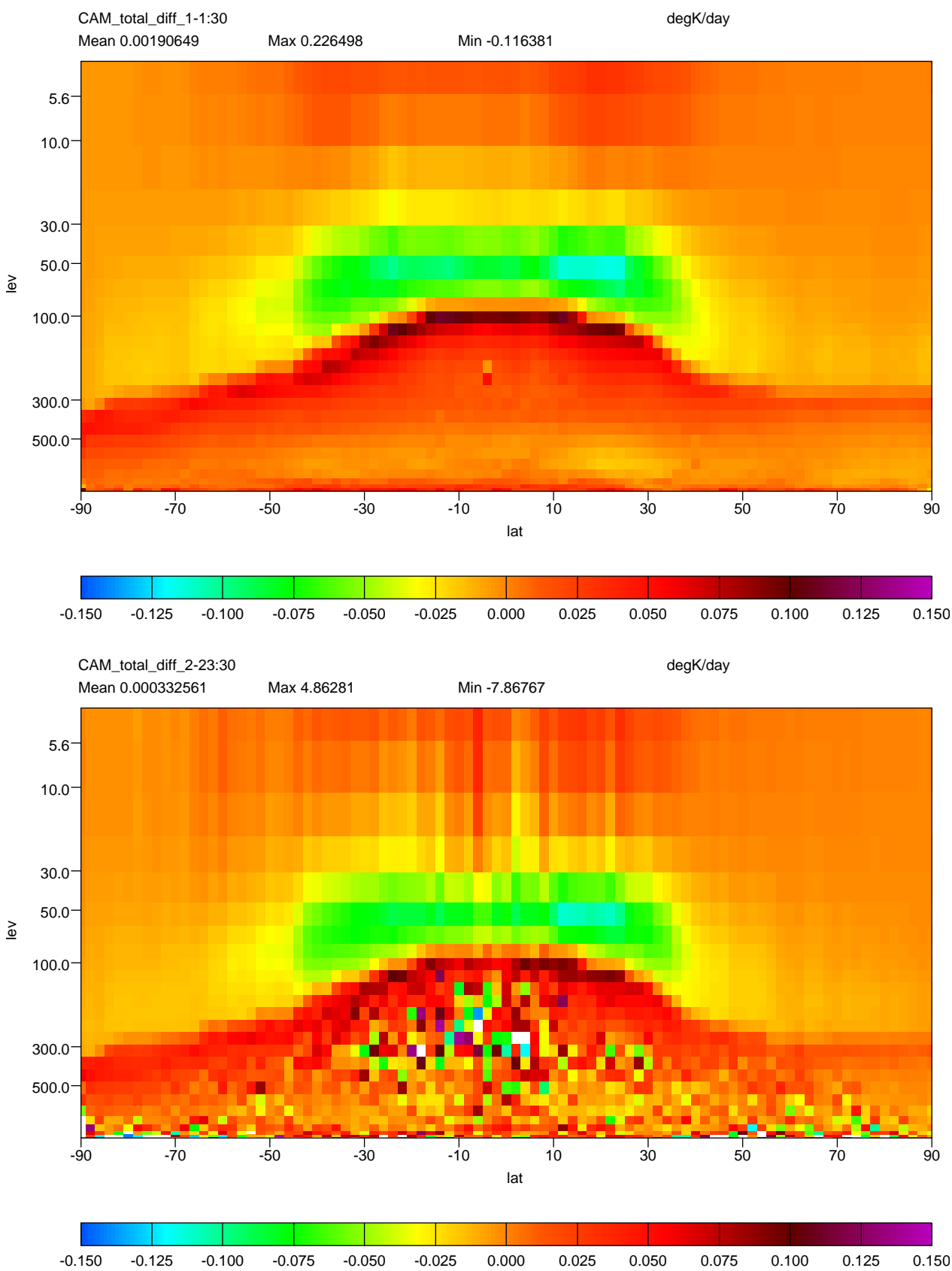

Figure 9: Difference between with/without tropospheric ozone CAM3 total heating rates, at the beginning of the two-day run (top), and at the end (bottom). 



Figure 10: Difference between heating rate differences due to tropospheric ozone obtained from IMPACT and CAM. Top: the beginning of day 1. Bottom: end of day 2 . 

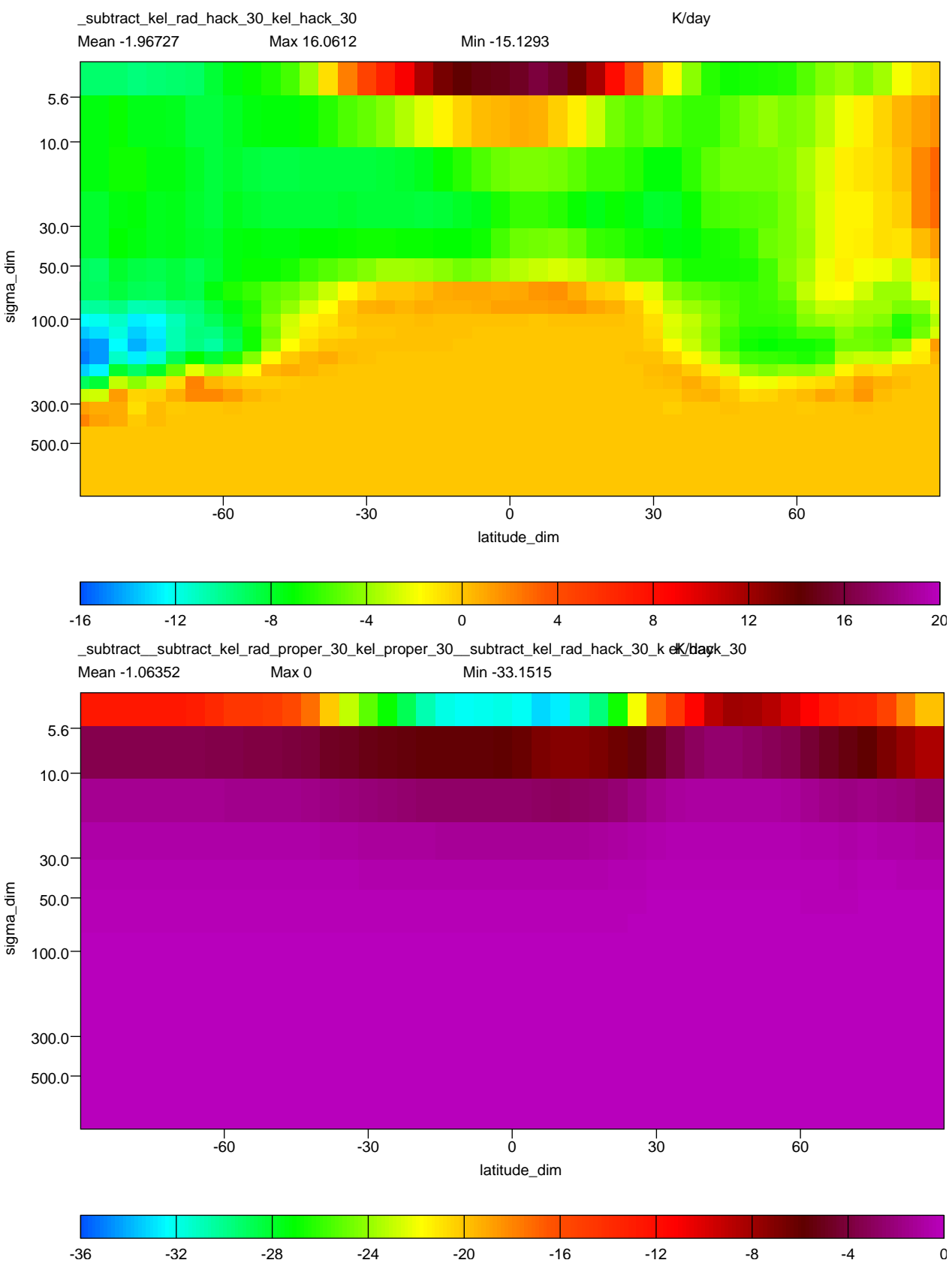

Figure 11: Top: kel_rad - kel when the top layer heating rates were forced to be equal to the one lower layer. Bottom: the difference between the top plot and an equivalent run with an unaltered radiation code. Both are means for day 30. Since IMPACT has a cold bias, a negative value in the lower plot indicates an improvement. 